\title{
338 - Risk factors for moderate cognitive impairment and dementia in the Kazakh and Kyrgyz populations (preliminary results) \\ Saule T.Turuspekova ${ }^{1}$, B. Demesinova ${ }^{1}$, Z.Rayimzhanov', R. Nurzhanova ${ }^{1}$, L. Bespalova ${ }^{3}$, I. Murkamilov ${ }^{4}$, A.Arykova $^{5}$.
}

${ }^{1}$ Kazakh National Medical University, Department of Nervous Diseases with a course of neurosurgery, Almaty, Kazakhstan. ${ }^{2}$ Kyrgyz State Medical Academy, Department of Public Health, Bishkek, Kyrgyzstan, ${ }^{3}$ Kazakh National Medical University, Department of Psychiatry and Narcology. Almaty, Kazakhstan. ${ }^{4}$ Kyrgyz State Medical Academy, Department of Faculty Therapy, Bishkek, Kyrgyzstan. ${ }^{5}$ Kyrgyz-Russian Slavic University, Department of Therapy, Bishkek, Kyrgyzstan,

The current demographic situation in Kazakhstan and Kyrgyzstan is associated with pronounced processes of population aging. Today, about two million elderly people live in Kazakhstan, which makes up more than $10 \%$ of the population, which crosses a seven percent threshold for determining the "aging" country in the world.

For Kyrgyzstan, an increase in the proportion of the elderly is a new phenomenon. At the beginning of 2016, there were 965,385 people over 50 in Kyrgyzstan, which is $16 \%$ of the total population, of whom 60 and over are $7.06 \%$. According to the average version of the forecast, the relative number of people 60 years and older will almost triple by 2050. (M. B. Denisenko, 2011, Report of a statistical study, 2017) The aging of the population is the most significant social problem of the XXI st century and is associated with the development of cognitive impairment and dementia. Moderate cognitive impairment, according to several authors are an intermediate stage between normal aging and dementia (Brodaty $\mathrm{H}$., 2013, Peterson, 2009).

The cause of cognitive disorders in old age can be a number of diseases, including neurodegenerative. According to projections, it is expected that neurodegenerative diseases will surpass cancer as the leading cause of death by 2040 (Walter U., 2013). The Global prevalence pattern of dementia depends on several factors, from life expectancy to the health status, last but not least from the particular environment.

Purpose of the study. To study the prevalence of cognitive impairment and the main risk factors for their occurrence among people over 60 in the Kazakh and Kyrgyz populations.

Materials and methods. The screening was attended by 300 respondents (150 Kazakhs and $150 \mathrm{Kyrgyz})$ aged 60 to 90 years (average age 65.6). The material was collected using the Questionnaire for the Champ Clinic and the MOCA test (cut-off point <26) based on the city polyclinics of Almaty and Bishkek.

Results: Results: moderate cognitive impairment was detected - 39.9\%, mild cognitive impairment $33.4 \%$, normal cognitive function $-26.7 \%$ in Kazakhs. In the Kyrgyz population, a moderate cognitive impairment was identified - 48.3\%; pronounced cognitive impairment - 15.4\%, dementia - $8.2 \%$. Expected risk factors for cognitive impairment: 1 ) in Kazakhs, arterial hypertension $-86.89 \%$, cardiac ischemia, angina pectoris $-70.21 \%$, pathology of the thyroid gland and pancreas (diabetes, hyperthyroidism and hypothyroidism) - $29.50 \%$, atherosclerotic vascular diseases $-29.9 \%$, brain injury $18.22 \%$, education level $-4.24 \%$, depression $-0.6 \%$, respectively.

2 ) in Kyrgyz: arterial hypertension - 49\%, cardiac ischemia, angina pectoris - $13 \%$, pathology of the thyroid gland and pancreas (diabetes, hyperthyroidism and hypothyroidism) - $21 \%$, low level of education - $11 \%$, Bad habits of smoking and alcohol - $25 \%, 20 \%$, respectively.

Conclusions: The transformation of the demographic burden can have important implications for the 
social security system.We have an urgent need to continue screening the population to identify the main risk factors for moderate cognitive impairment in individuals in the Kazakh and Kyrgyz populations. 